\title{
MODELO DE DESARROLLO SUSTENTABLE PARA LA INDUSTRIA DE RECUBRIMIENTOS CERÁMICOS
}

\author{
Jorge CARRO SUÁREZ ${ }^{1,2 *}$, Bernardo REYES GUERRA ${ }^{3}$, Genoveva ROSANO ORTEGA², \\ Jaime GARNICA GONZÁLEZ ${ }^{4}$ y Beatriz PÉREZ ARMENDÁRIZ ${ }^{2}$
}

${ }^{1}$ Universidad Politécnica de Tlaxcala. Avenida Universidad Politécnica 1, San Pedro Xalcaltinco, Tepeyanco, Tlaxcala, México, C. P. 90180

${ }^{2}$ Universidad Popular Autónoma del Estado de Puebla. Avenida Juárez esquina con Calle 23 Sur 2301, Zona Esmeralda, Puebla, Puebla, México, C. P. 72060

${ }^{3}$ Instituto Tecnológico y de Estudios Superiores de Monterrey, Campus Puebla. Vía Atlixcáyotl 2301, Reserva Territorial Atlixcáyotl, Puebla, Puebla, México, C. P. 72453

${ }^{4}$ Universidad Autónoma del Estado de Hidalgo. Edificio Torres de Rectoría, Carretera Pachuca-Actopan, kilómetro 4.5, 7o. Piso, Colonia Campo de Tiro, Pachuca de Soto, Hidalgo, México, C. P. 42039

*Autor para correspondencia: jorge.carro@uptlax.edu.mx

(Recibido agosto 2015; aceptado marzo 2016)

Palabras clave: factores de sustentabilidad, sustentabilidad empresarial, industria cerámica

\section{RESUMEN}

La sociedad del siglo XXI requiere la incorporación del desarrollo sustentable en su vida diaria, cuyo proceso integral exige concebirlo no únicamente como un esfuerzo ecológico, también debe considerar otros componentes de orden social, económico e institucional para un modelo que contribuya al enriquecimiento de la calidad de vida. Diversos estudios han identificado que las compañías no desarrollan una estrategia de sustentabilidad porque no es su prioridad, no tienen dominio del tema y se presenta una disyuntiva entre ser sustentables o rentables. El objetivo de esta investigación es proponer un modelo de desarrollo sustentable identificando qué factores de sustentabilidad promueven las empresas y cómo las beneficiarían si los enfocan hacia el reconocimiento como sustentables. Dada su influencia económica, el sector en estudio es el de recubrimientos cerámicos en México, en particular empresas del estado de Tlaxcala. Una primera etapa fue analizar diferentes modelos de sustentabilidad publicados e implementados por la industria para identificar sus puntos de intersección. En una segunda etapa, se realizó un diagnóstico de la percepción por medio de un instrumento de evaluación (Alfa de Cronbach 0.927). Los resultados obtenidos revelan una percepción promedio significativa $(\mathrm{p}<0.05)$ en la seguridad y salud en el trabajo $(4.40)$ con tendencia hacia muy determinante mientras la ciencia para el desarrollo sustentable (3.90) e integración ambiental (3.21) presentan una tendencia hacia moderadamente determinante, con lo que se concluye que es necesario que las empresas consideren estos factores para que sean reconocidas y obtener así una certificación integral como empresa sustentable.

Key words: factors of sustainability, business sustainability, tile industry 


\begin{abstract}
Society of the twenty-first century requires the incorporation of sustainable development into its daily life, this integral process requires conceiving it not only as an ecological effort but we must also consider other social, economic and institutional components for a model that will contribute to the enrichment of the quality of life. Various studies have identified that companies do not develop a sustainability strategy because it is not their priority. In addition, they do not know enough about the topic and also they have the dilemma of being either sustainable or profitable. The objective of this research is to propose a model of sustainable development by identifying which factors of sustainability are promoted by companies and how these companies benefit from being recognized as sustainable companies. Due to its economic influence, the sector under study is the ceramic coatings sector in Mexico, specifically considering the companies located in the state of Tlaxcala. In a first approach, an analysis of different models of sustainability published and implemented by the industry was performed in order to identify their points of intersection. In a second phase, an assessment of perception was carried out through the evaluation instrument of Cronbach's alpha 0.927. The results revealed a significant average perception $(\mathrm{p}<0.05)$ regarding safety and health at work (4.40) presenting a trend toward very decisive, while science for sustainable development (3.90) and environmental integration (3.21) presented a rather decisive trend, concluding that companies need to consider these factors to be recognized and certified as a sustainable company.
\end{abstract}

\section{INTRODUCCIÓN}

El término de desarrollo sustentable (DS) nace con la idea de administrar de forma eficiente y racional los recursos con los que cuenta la sociedad, de tal manera que sea posible mejorar el bienestar de la población actual sin comprometer la calidad de vida de las generaciones futuras (UN 1987). Asimismo, se le considera una megatendencia emergente que muchas empresas visualizan como un desafío que puede afectar su competitividad al no percibir su carácter estratégico (Lubin y Esty 2010). Por lo que alcanzar un crecimiento económico que conserve y enriquezca en vez de destruir las bases naturales en las que inevitablemente se asienta la actividad humana, constituye el objetivo principal de cualquier estrategia de sustentabilidad (Calva 2007).

En la actualidad, el DS es un tema que está cobrando mayor interés en las empresas de todo el mundo y especialmente, en las de México. Sin embargo, es importante destacar que no se debe ver como simple filantropía, sino como una nueva forma de analizar el desempeño de la organización desde cuatro perspectivas diferentes y complementarias: la económica (relativa a la necesidad de que la empresa sea rentable para perdurar en el tiempo), la social (para atender los impactos tanto internos como externos de esta índole que pudieran presentar las operaciones de la empresa), la ambiental (para cuidar el impacto que pudiera ejercer la operación de la empresa sobre el ambiente y los recursos naturales) y la institucional (para fomentar su desarrollo como parte de la cultura empresarial).

Diversas investigaciones han empezado a identificar las relaciones entre estas dimensiones, como el concepto de ecoeficiencia, que busca unir las mejoras ambientales y económicas (Rincón y Wellens 2011), la relación social y ambiental a través de la educación (Avilés et al. 2014) o como parte fundamental de la cultura en las Instituciones de Educación Superior (Juárez-Nájera et al. 2010, Espinosa et al. 2013).

No obstante, a pesar de estos esfuerzos, todavía existen empresas que no han permeado totalmente el concepto de DS al interior de sus organizaciones, siendo una de las principales causas el desconocimiento del tema seguido por la falta de recursos asignados para el mismo al no ser considerado como una prioridad (KPMG 2011). En México, hay empresas que han tomado la iniciativa de empezar una cultura de sustentabilidad, pero que aún la visualizan como un cambio exclusivo en la producción. Lo ideal es que sea parte de su estrategia, para que así, puedan involucrar al resto de su organización en la búsqueda de mejorar sus niveles de productividad y por consecuencia, también su rentabilidad (López 2012).

Con base en lo anterior, la presente investigación tiene como objetivo proponer un modelo de DS determinando qué factores de sustentabilidad son significativos para las empresas y enfocarlas hacia 
un reconocimiento como empresas sustentables a través del análisis de diversos modelos teóricos y empresariales, así como de diversas normas de certificación. Para tal fin, se seleccionó a la industria de los recubrimientos cerámicos en México, por ser un sector poco estudiado pero de gran trascendencia económica al ubicarse entre los diez principales productores a nivel mundial (Stock 2014). El caso de estudio fue el estado de Tlaxcala que ocupa el segundo lugar a nivel nacional con seis empresas de recubrimientos cerámicos instaladas y en operación (Sociedad Mexicana de Cerámica Zona Norte 2014).

\section{ANTECEDENTES}

\section{Modelos y factores de DS}

En la década de los 90, el concepto de DS alcanzó su mayor difusión, constituyéndose en una moda. A fines del siglo XX su popularidad implicó que su presencia se distinguiera en todas las estructura sociales, como una fórmula que sin duda, nos llevaría a alcanzar una mejor calidad de vida. Sin embargo, este proceso se ha desgastado, se recurre con insistencia a la sustentabilidad pero difícilmente se le define como un concepto destinado a la acción colectiva en un grupo social. De ahí nace la necesidad de identificar factores e indicadores que sirvan para medir la distancia y el sentido de la variación de un modelo entre el estado inicial y el estado de transición, enfocado hacia un escenario sustentable de desempeño de la sociedad (Achkar 2005). En este sentido, la comisión Brundtland reconoció que el concepto de DS debe ir más allá que el aspecto ambiental, también debe considerar el bienestar humano, el bienestar ecológico y sus interacciones. Esta perspectiva dio lugar a la publicación del llamado "Libro azul" en el que se presentaron cuatro dimensiones de sustentabilidad: económica, social, ambiental e institucional (Durán 2000).

Asimismo, Achkar (2005) estableció que la sustentabilidad se puede fundamentar en cuatro dimensiones que interactúan entre sí, a las cuales denominó dimensión físico-biológica, social, económica y política. Dimensiones que presentan una estrecha relación con las presentadas por el Informe Brundtland.

Este enfoque, planteado y utilizado por diversos países e instituciones hace énfasis en desarrollar capital económico y capital social mientras se ejerce una sólida administración del capital ambiental (Hoegen 2009). Bajo esta nueva visión, se observó que la sustentabilidad puede integrar por lo menos a tres factores principales que son el ambiente, la economía y la sociedad (Salcedo et al. 2010, Mihelcic y Zimmerman 2012). Como resultado, a través de los años han proliferado teorías, modelos e indicadores que con el tiempo han evolucionado. No obstante, todos presentan aspectos en común.

Entre los modelos más reconocidos destacan los siguientes:

Modelo Brundtland: en su dimensión social se destaca la salud, educación, equidad, vivienda y seguridad. En la dimensión económica considera al desarrollo económico como principal factor y en la dimensión ambiental el cuidado del aire, suelos y agua (UN 1987).

Modelo del servicio estadístico gubernamental del Reino Unido (GSS, por sus siglas en inglés): en la dimensión social considera prioritario la salud, el fomento de las destrezas, la motivación, el conocimiento y el espíritu. En la parte económica destaca la atención hacia los edificios y maquinaria, y en la dimensión ambiental prioriza el cuidado y buen manejo de los minerales, bosques, flora, fauna, agua, aire y suelos (GSS 2004).

El modelo de tetraedro de Achkar: establece que las buenas relaciones entre clases sociales es el principal factor de la dimensión social. También considera a las relaciones de propiedad y circuito de finanzas en la dimensión económica y por último, las relaciones del uso y manejo de los bienes de la naturaleza en beneficio de la humanidad (Achkar 2005).

Modelo Barber-Zapata: entrelaza las dimensiones ecológica, económica y social en una zona común que es donde se logra el DS, como un indicador de que las tres dimensiones se están realizando. Esto quiere decir, que si alguna área llega a omitirse, se tendría otro tipo de desarrollo (Salcedo et al. 2010).

Con estos principios, las empresas en México han diseñado sus propios modelos. Sin embargo, las nuevas tendencias en el siglo XXI exigen el fortalecer e impulsar a la sustentabilidad no sólo en la parte ambiental y social, que es donde se concentran la mayoría de los esfuerzos. Tal es el caso del sector de recubrimientos cerámicos en México, en el que sus modelos de DS se enfocan principalmente, en la dimensión ambiental y social, por lo que sus operaciones sólo las han llevado a ser reconocidas como empresas limpias o como socialmente responsables. A pesar de que realizan acciones que impactan en otros aspectos de sustentabilidad y que requieren ser reconocidas. De tal forma, que para complementar y fortalecer la propuesta de factores de sustentabilidad, también se analizaron las normas y criterios de certificación y reconocimiento del cuadro I. 
CUADRO I. NORMAS Y CRITERIOS REFERENTES A LA SUSTENTABILIDAD EMPRESARIAL

\begin{tabular}{|c|c|}
\hline Nombre de la norma & Que hace referencia a: \\
\hline $\begin{array}{l}\text { Organización Internacional de } \\
\text { Estandarización } \\
\text { (ISO, por sus siglas en inglés) } \\
\text { ISO } 14001\end{array}$ & $\begin{array}{l}\text { Sistema de certificación ambiental que reconoce la implantación de un sistema de gestión que } \\
\text { promueve la protección ambiental y la prevención de la contaminación desde un punto de } \\
\text { vista de equilibrio con los aspectos socioeconómicos de acuerdo con la Asociación Española } \\
\text { de Normalización y Certificación (AENOR 2014). }\end{array}$ \\
\hline ISO 14006 & $\begin{array}{l}\text { Gestión de ecodiseño. Demuestra que la organización ha adoptado un sistema de gestión para } \\
\text { identificar, controlar y mejorar de manera continua los aspectos ambientales de los productos } \\
\text { y/o servicios, facilitando información a los clientes sobre sus acciones y productos en búsqueda } \\
\text { de mejoras ambientales (AENOR 2014). }\end{array}$ \\
\hline Empresa limpia & $\begin{array}{l}\text { Certificación emitida por la Procuraduría Federal de Protección al Ambiente en México (PRO- } \\
\text { FEPA) que consiste en una serie ordenada de actividades que buscan hacer más eficientes los } \\
\text { procesos productivos de una empresa a través de la reducción del consumo de agua, energía } \\
\text { y generación de residuos (PROFEPA 2014). }\end{array}$ \\
\hline Bolsas de valores sustentables & $\begin{array}{l}\text { A través del reconocimiento por el índice mundial de sustentabilidad Dow Jones (DJSI, por } \\
\text { sus siglas en inglés), se monitorea el desempeño de las compañías que cotizan en las bolsas } \\
\text { de valores en sus indicadores sobre aspectos económicos, sociales, ambientales y de gobierno } \\
\text { corporativo (DJSI 2014). }\end{array}$ \\
\hline ISO 26000 & $\begin{array}{l}\text { Responsabilidad social. Certificación que ayuda a cumplir y operar de manera responsable } \\
\text { el cumplimiento de los requisitos que la sociedad exige fundamentada en siete materias: go- } \\
\text { bernanza de la organización, derechos humanos, prácticas laborales, ambiente, prácticas de } \\
\text { justa operación, asuntos de consumidores y participación activa y desarrollo de la comunidad } \\
\text { (GlobalSTD 2014). }\end{array}$ \\
\hline $\begin{array}{l}\text { Empresa socialmente responsable } \\
\text { (ESR) }\end{array}$ & $\begin{array}{l}\text { Emitida por el Centro Mexicano de Filantropía (Cemefi) que comprende cuatro líneas que } \\
\text { explican su presencia en toda actividad de la empresa: ética y gobernabilidad empresarial, } \\
\text { calidad de vida en la empresa, vinculación y compromiso con la comunidad y su desarrollo, } \\
\text { así como el cuidado y preservación del ambiente (Cemefi 2014). }\end{array}$ \\
\hline $\begin{array}{l}\text { Instituto Nacional Estadounidense } \\
\text { de Estándares } \\
\text { (ANSI, por sus siglas en inglés) } \\
\text { ANSI-A138.1 }\end{array}$ & $\begin{array}{l}\text { Estándar voluntario que resume los criterios ambientales y sociales para azulejos cerámicos } \\
\text { sostenibles, emitida por el Consejo Cerámico de Norteamérica (TCNA, por sus siglas en } \\
\text { inglés). Su certificación es reconocida como cuadrado verde (green squared), indicando que } \\
\text { los productos cubren criterios ambientales, gestión final de la vida del producto, gobierno } \\
\text { corporativo e innovación (TCNA 2014). }\end{array}$ \\
\hline Ley del Seguro Social & $\begin{array}{l}\text { Ley que establece que la seguridad social tiene la finalidad de garantizar el derecho a la salud, } \\
\text { la asistencia médica, la protección de los medios de subsistencia y los servicios sociales ne- } \\
\text { cesarios para el bienestar individual y colectivo por parte del Instituto Mexicano del Seguro } \\
\text { Social (IMSS) en México (IMSS 2014). }\end{array}$ \\
\hline $\begin{array}{l}\text { Normas Oficiales Mexicanas de } \\
\text { la Secretaría del Trabajo y } \\
\text { Previsión Social }\end{array}$ & $\begin{array}{l}\text { Conjunto de normas oficiales mexicanas en seguridad y salud en el trabajo, en la que la Se- } \\
\text { cretaría de Trabajo y Previsión Social (STPS) en México fundamenta como tarea primordial } \\
\text { el estudiar y ordenar las medidas de seguridad e higiene industriales, para la protección de } \\
\text { los trabajadores y vigilar su cumplimiento (STPS 2014) }\end{array}$ \\
\hline
\end{tabular}

Fuente: Elaboración propia

Lo anterior, revela la necesidad de un modelo que identifique realmente qué factores de sustentabilidad impactan en cada dimensión del DS para promover nuevas estrategias, especialmente, en la dimensión económica para que el desarrollo ecológico sea rentable. Así en consecuencia, tendrá una mejor aceptación por los empresarios, así como en la dimensión institucional, para la creación y fomento de una nueva cultura organizacional.

\section{MATERIALES Y MÉTODOS}

\section{Diseño de la investigación}

Para el presente trabajo se planteó una investigación no experimental, descriptiva, cuantitativa y transversal. El análisis buscó especificar las características y perfiles de un objeto de estudio para proponer un modelo de DS (Hernández et al. 2010). El objeto de estudio fue el sector de recubrimientos 
cerámicos en México, por ser un sector poco estudiado en materia de DS pero reconocido por su productividad al ubicarse en el décimo lugar de países productores a nivel mundial (Stock 2014). Por su productividad destacan tres grandes fabricantes: Lamosa Revestimientos de Grupo Lamosa S.A.B. de C.V. con once plantas instaladas, Cerámica Vitromex de Grupo Industrial Saltillo S.A.B. de C.V con cinco plantas y una de Interceramic de Grupo Internacional de Cerámica S.A.B. de C.V., con una capacidad instalada entre las tres empresas de 216.4 millones de $\mathrm{m}^{2}$ al año, lo que representa el $95 \%$ de la producción total del país (Stock 2014). Asimismo, el estado de Tlaxcala es reconocido por ocupar el segundo lugar en cantidad de empresas instaladas y en operación en el país.

\section{Instrumento de evaluación}

Después de analizar los modelos teóricos, empresariales y diversas normas de certificación y reconocimiento, se diseñó un cuestionario de 42 reactivos para identificar la percepción que actualmente tienen las empresas sobre los factores e indicadores propuestos para las dimensiones de DS.

Para su aplicación se consideró dentro del perfil del encuestado al personal que tuviera experiencia en el sector de al menos dos años en puestos de mando medio o directivo. La encuesta fue validada con una muestra $n=20$ a través del coeficiente de alfa de Cronbach.

Para cada pregunta se aplicó una medida métrica en la modalidad de medición por intervalos, manteniendo un orden determinante entre categorías iguales (Hernández et al. 2010). De esta forma, para medir las escalas en cada ítem se aplicó el método de escalamiento Likert. Se consideró una escala de cinco categorías de respuesta con unidades métricas del 1 al 5, en donde el 1 se especifica como nada determinante, 2 poco determinante, 3 moderadamente determinante, 4 muy determinante y 5 extremadamente determinante (Vagias 2006). Con lo anterior se midió la percepción sobre los factores de DS propuestos, entendiendo el concepto determinante como la acción que influye para tomar una decisión (Garzón 1995). Finalmente se aplicó el instrumento en las empresas de cerámica instaladas en el estado de Tlaxcala.

\section{Análisis estadístico}

Para establecer las diferencias significativas entre el nivel de determinación de los factores de DS, se aplicó un análisis de varianza para un modelo completo de bloques aleatorizados (Montgomery 2014). Asimismo, se empleó la prueba de Tukey para realizar comparaciones múltiples con un nivel de significancia del $95 \%$. Los resultados fueron analizados con el programa Minitab 16®.

\section{RESULTADOS Y DISCUSIÓN}

\section{Factores de sustentabilidad}

Con base en el análisis teórico, en las acciones que realizan las empresas de cerámica en las dimensiones social, económica, ambiental e institucional y en las normas y requisitos para lograr una certificación y/o reconocimiento, en el cuadro II se presenta la propuesta de factores de sustentabilidad para el sector de recubrimientos cerámicos en México. Adicionalmente, se presentan los sub-factores y líneas de acción, que formaron la base para el diseño del instrumento.

El instrumento diseñado se aplicó en cinco empresas de recubrimientos cerámicos del estado de Tlaxcala, lo que representó el $83.33 \%$ del total de la muestra recabando 20 casos.

La confiabilidad se calculó con el coeficiente de alfa de Cronbach, ésta resultó ser de 0.927, lo que indica una elevada confiablidad (Hernández et al. 2010)

En el cuadro III se observa que el factor seguridad y salud en el trabajo, representado por la letra "a" presenta una diferencia significativa $(\mathrm{p}<0.05)$, además de ser el que muestra la media más alta (4.40), ubicándose entre un nivel muy determinante y extremadamente determinante. La anterior tendencia es debida principalmente a la importancia de contar con prestaciones para el cuidado de la salud de los trabajadores y con seguridad social para la familia tal como lo indica la ley del Seguro Social y las normas oficiales de la Secretaría del Trabajo y Previsión Social en México. Los factores ciencia para el DS (3.90) e integración ambiental (3.21) representados por las letras "b y c" respectivamente, también presentaron diferencias significativas, ubicándose entre moderadamente determinante y muy determinante. Debido en su mayoría, a la poca relevancia que se le da al participar en conjunto con universidades y centros de investigación en proyectos enfocados hacia el DS, así como el trabajar en conjunto con cámaras de comercio y con los legisladores.

En lo que se refiere a los factores restantes representados como "ab", no presentaron diferencias significativas, concentrándose en una actitud favorable positiva.

Finalmente y con base en los resultados obtenidos por el instrumento, se propone el modelo de DS para el sector de recubrimientos cerámicos (Fig. 1). En este sector, se destacan las cuatro dimensiones 
CUADRO II. FACTORES, SUBFACTORES Y LÍNEAS DE ACCIÓN EN MATERIA DE SUSTENTABILIDAD DE LA INDUSTRIA CERÁMICA EN MÉXICO

\begin{tabular}{|c|c|c|}
\hline $\begin{array}{l}\text { Factor de } \\
\text { sustentabilidad }\end{array}$ & Subfactor & Líneas de acción \\
\hline \multirow{3}{*}{$\begin{array}{l}\text { Administración de los } \\
\text { recursos humanos }\end{array}$} & Equidad & $\begin{array}{l}\text { Desarrollo de oportunidades de formación para garantizar la igualdad de } \\
\text { crecimiento profesional para hombres y mujeres }\end{array}$ \\
\hline & Motivación laboral & $\begin{array}{l}\text { Retención de personal con talento, con compromiso y destacado } \\
\text { Promociones para mejorar el salario }\end{array}$ \\
\hline & $\begin{array}{l}\text { Inversiones en el capital } \\
\text { humano }\end{array}$ & Programas de capacitación e incentivos de productividad para el personal \\
\hline \multirow{3}{*}{$\begin{array}{l}\text { Seguridad y salud en } \\
\text { el trabajo }\end{array}$} & Salud & $\begin{array}{l}\text { Prestaciones para el cuidado de la salud de los trabajadores } \\
\text { Seguridad social }\end{array}$ \\
\hline & Seguridad e higiene & $\begin{array}{l}\text { Programas de prevención de accidentes } \\
\text { Integración de comisiones mixtas de seguridad e higiene }\end{array}$ \\
\hline & $\begin{array}{l}\text { Actividades deportivas y } \\
\text { recreativas }\end{array}$ & $\begin{array}{l}\text { Desarrollo de torneos deportivos entre el personal } \\
\text { Convivencias entre el personal en fechas especiales para la empresa }\end{array}$ \\
\hline \multirow{2}{*}{$\begin{array}{l}\text { Responsabilidad social } \\
\text { corporativa }\end{array}$} & Valores sociales y éticos & $\begin{array}{l}\text { Implantación de un código de ética } \\
\text { Líneas de transparencia para evitar actos de corrupción } \\
\text { Rechazo a las prácticas discriminatorias }\end{array}$ \\
\hline & Programas sociales & $\begin{array}{l}\text { Apoyo a instituciones de carácter social y educativo } \\
\text { Proyectos de voluntariado en las comunidades } \\
\text { Inversiones en favor de las comunidades y su entorno }\end{array}$ \\
\hline
\end{tabular}

Inversión para disminuir el Programas de administración y control de insumos

consumo de energía Programas de optimización de procesos

Sistemas de alumbrado inteligente

Inversión verde

Implementación de sistemas de energía renovable

Inversión en tecnología Adopción de tecnología de vanguardia para el desarrollo de líneas de productos ambiental dirigidas al cuidado del ambiente

Actualización de equipos de transmisión de potencia electromecánicos por otros de mayor eficiencia para disminuir el consumo de energéticos

Control de emisiones

Implementación de programas internos para la vigilancia del cumplimiento de los reglamentos ambientales aplicables

Realización de auditorías ambientales de forma periódica

Control de emisión de $\mathrm{CO}_{2}$ a través de estudios de emisiones a la atmósfera para disminuir el impacto al medio

Prevención de la contaminación

Manejo de residuos sólidos

Estandarización de las prácticas de recuperación de materiales a través de procedimientos de control interno encaminados a uniformizar los pasos requeridos para confinar los distintos materiales recuperados y asegurar su adecuado tratamiento

\begin{tabular}{lll} 
& Reciclado de productos & Programa de reciclado de papel, cartón, madera, PET y aluminio \\
\cline { 2 - 3 } $\begin{array}{l}\text { Gestión de recursos } \\
\text { naturales }\end{array}$ & $\begin{array}{l}\text { Tratamiento y calidad del } \\
\text { agua }\end{array}$ & $\begin{array}{l}\text { Programas de tratamiento de aguas } \\
\text { Control de reciclado de agua para servicios generales dentro del proceso } \\
\text { Operación de prácticas para reducir el consumo }\end{array}$ \\
\cline { 2 - 3 } & Áreas verdes & $\begin{array}{l}\text { Fomento y cuidado de áreas verdes dentro y fuera de las instalaciones } \\
\text { Programas de reforestación }\end{array}$ \\
\hline \multirow{2}{*}{$\begin{array}{l}\text { Integración ambiental } \\
\text { Estrategias de desarrollo } \\
\text { sustentable }\end{array}$} & $\begin{array}{l}\text { Diseño de líneas de productos sustentables } \\
\text { Monitoreo de la organización, del desempeño y de las acciones sustentables } \\
\text { Participación en las políticas públicas }\end{array}$ \\
\hline
\end{tabular}

Fuente: Elaboración propia. 
CUADRO II. FACTORES, SUBFACTORES Y LÍNEAS DE ACCIÓN EN MATERIA DE SUSTENTABILIDAD DE LA INDUSTRIA CERÁMICA EN MÉXICO

\begin{tabular}{lll}
\hline $\begin{array}{l}\text { Factor de } \\
\text { sustentabilidad }\end{array}$ & Subfactor & Líneas de acción \\
\hline $\begin{array}{l}\text { Transparencia en la } \\
\text { información }\end{array}$ & Acceso a la información & Presentación de informes \\
\hline $\begin{array}{l}\text { Ciencia para el } \\
\text { desarrollo sustentable }\end{array}$ & $\begin{array}{l}\text { Gastos en investigación y } \\
\text { desarrollo experimental }\end{array}$ & $\begin{array}{l}\text { Inversión y convenios con instituciones de educación superior } \\
\text { Creación de centros de investigación y desarrollo }\end{array}$ \\
\hline
\end{tabular}

Fuente: Elaboración propia.

CUADRO III. NIVEL DE DETERMINACIÓN Y RECONOCIMIENTO DE LOS FACTORES DE DESARROLLO SUSTENTABLE (DS) PROPUESTOS

\begin{tabular}{lcl}
\hline Factor de DS & Nivel de determinación ${ }^{1}$ & Aplicado por: \\
\hline Seguridad y salud en el trabajo & $4.40 \pm 0.20 \mathrm{a}$ & Ley del Seguro Social y STPS \\
Administración de los recursos humanos & $4.34 \pm 0.17 \mathrm{a}, \mathrm{b}$ & - \\
Inversión verde & $4.29 \pm 0.17 \mathrm{a}, \mathrm{b}$ & Bolsa de Valores Sustentable \\
Prevención de la contaminación & $4.19 \pm 0.14 \mathrm{a}, \mathrm{b}$ & PROFEPA Empresa Limpia \\
Gestión de los recursos naturales & $4.17 \pm 0.15 \mathrm{a}, \mathrm{b}$ & Norma ISO 14001 \\
Transparencia de la información & $4.00 \pm 0.24 \mathrm{a}, \mathrm{b}$ & Cemefi ESR \\
Responsabilidad social corporativa & $4.00 \pm 0.17 \mathrm{a}, \mathrm{b}$ & Cemefi ESR \\
Ciencia para el desarrollo sustentable & $3.90 \pm 0.24 \mathrm{~b}$ & - \\
Integración ambiental & $3.21 \pm 0.17 \mathrm{c}$ & Normas ANSI A138.1 e ISO 14006 \\
\hline
\end{tabular}

${ }^{1}$ Los valores con letras iguales no mostraron diferencias significativas $(\mathrm{p}<0.05)$

STPS = Secretaría del Trabajo y Previsión Social, PROFEPA = Procuraduría Federal de Protección al Ambiente, ISO = Organización Internacional de Estandarización, ESR = Empresa Socialmente Responsable, ANSI = Instituto Nacional Estadounidense de Estándares

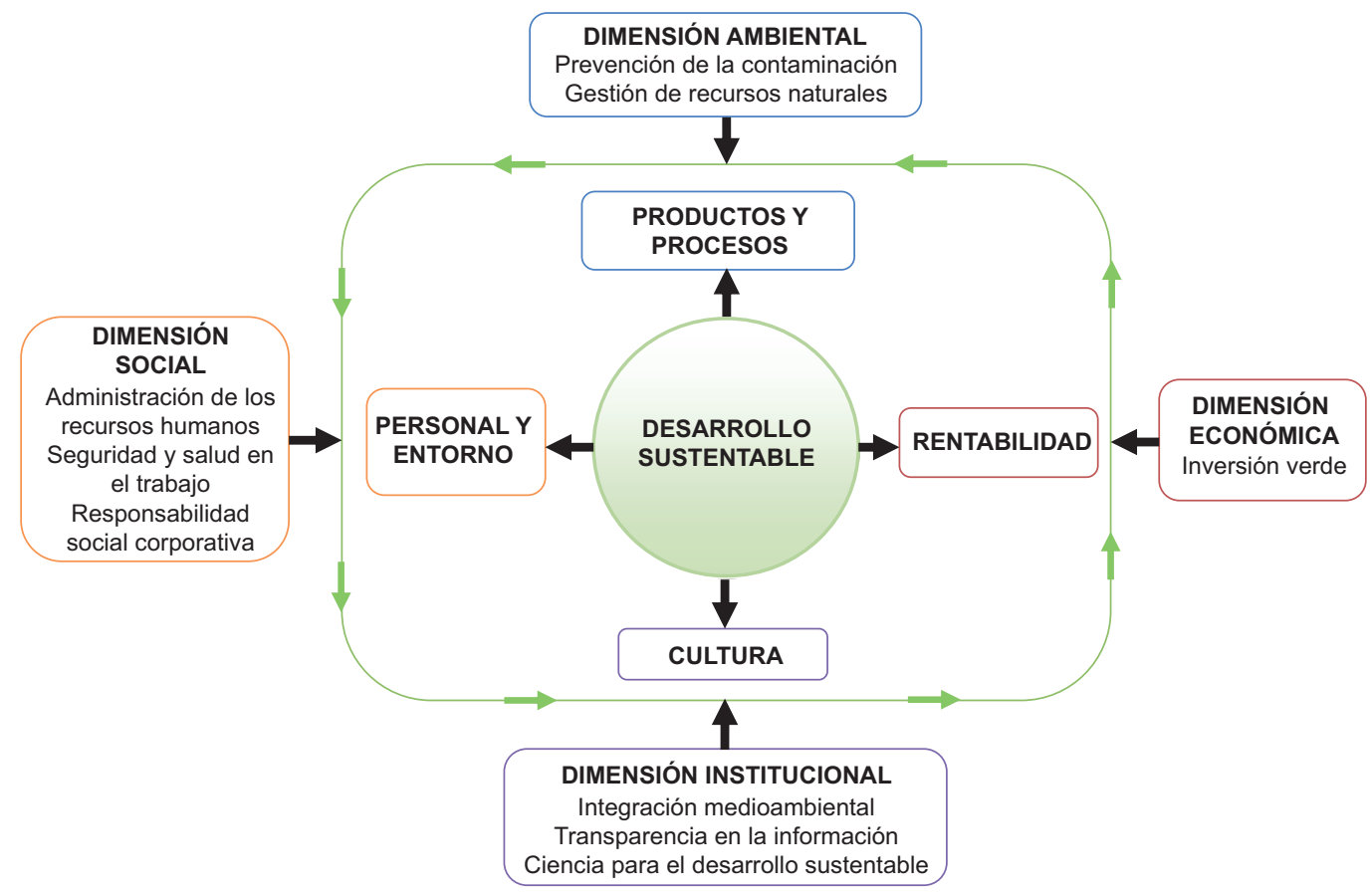

Fig. 1. Modelo de desarrollo sustentable para la industria de recubrimientos cerámicos Fuente: Elaboración propia 
de sustentabilidad: ambiental, social, económica e institucional con sus respectivos factores, enfocados hacia el objetivo de ser una empresa sustentable en productos, procesos, personal, entorno, rentabilidad y cultura.

\section{CONCLUSIONES}

En esta investigación se concluye que una empresa sustentable es aquella que realiza acciones de sustentabilidad en las dimensiones ambiental, social, económica e institucional.

Por lo que el modelo propuesto cumple con el objetivo de determinar qué factores de DS son significativos para las empresas, y que deben estar implícitos en su misión y visión, con el objetivo de enfocarse hacia el reconocimiento como empresas sustentables.

En el modelo se visualiza cómo las dimensiones de DS son determinantes en el proceso interno de la empresa, en el cual, todas ejercen influencia en los productos y procesos, en el personal y entorno, en la rentabilidad y en la cultura.

Así, en la dimensión ambiental se considera que los productos y procesos deben ser amigables con el ambiente a través de la prevención de la contaminación y de una buena gestión de sus recursos naturales. Adicionalmente, diseñar productos verdes, desde su materia prima hasta el final de su ciclo de vida. Basta identificar que desde hace años existe una creciente preocupación por el ambiente, la cual, se ha generalizado en las sociedades desarrolladas. Lo que ha constituido un nuevo entorno competitivo que no puede ser olvidado por la dirección de las empresas (Chamorro 2001).

En la dimensión social se busca el beneficio del personal y de su entorno con una eficiente administración de los recursos humanos. En este entorno se espera brindar salud, seguridad y crecimiento económico, tanto en la organización, como en el personal $\mathrm{y}$ en el lugar donde se ha establecido la empresa.

En lo que concierne a la dimensión económica, se plantea la propuesta de una rentabilidad tanto económica como social, en la búsqueda de generar resultados y/o beneficios con base en la inversión en tecnología para disminuir el consumo de energía y mejorar la calidad ambiental de los procesos. Se concibe además, que su objetivo no sólo sea generar ganancias económicas, sino también devolver algo a la sociedad a nivel social, económico y ambiental que le permita a la empresa un arraigo de forma permanente.
En la dimensión institucional se propone la creación de una cultura sustentable, con una misión y visión que impacte en todos sus actores, con normas, hábitos y valores que se practiquen dentro y fuera de la organización. Lo anterior impactará en una nueva forma de comportamiento y de educación (Dieleman y Juárez-Nájera 2008, Avilés et al. 2014).

Finalmente, toda acción realizada debe ser reconocida a través de un proceso de certificación, en el que se haga constar ante la sociedad a nivel nacional y mundial los beneficios económicos, ambientales y sociales que se están generando. Lo que refleja una mayor credibilidad al demostrar que son empresas que cumplen con normas establecidas por organismos nacionales e internacionales en cada dimensión.

Otro aspecto importante es el dar a conocer a las empresas que la sustentabilidad no está enfrentada con la rentabilidad. Lo anterior debido a que de acuerdo con la Bolsa Mexicana de Valores, en los últimos años se ha iniciado una tendencia por parte de los inversionistas en buscar compañías sustentables para invertir sus recursos en ellas, basándose en la premisa de que una empresa que sea sustentable generará valor a largo plazo y estará mejor preparada para lidiar con los retos económicos, sociales y ambientales que se presenten (Bibián 2014).

\section{REFERENCIAS}

Achkar M. (2005). Indicadores de sustentabilidad. Departamento de Geografía Facultad de Ciencias Universidad de la República Uruguay [en línea]. http:/www. ucipfg.com/Repositorio/MLGA/MLGA-03/semana2/ Indicadores_de_sostenibilidad.pdf 12/05/2015.

AENOR (2014). Certificación ambiental. Asociación Española de Normalización y Certificación [en línea]. http://www.aenor.com/aenor/certificacion/mambiente/ medio_ambiente.asp\#.Vti_QJPhCXo 01/06/2015.

Avilés K., Pérez B. y Rosano G. (2014). Relaciones entre variables sociales de aprendizaje en el desarrollo sustentable: El caso del Instituto Tecnológico de Tláhuac, D.F., México. Rev. Int. Contam. Ambie. 30, 407-416.

Bibián C. (2014). Empresas verdes ganan más dinero en la Bolsa Mexicana [en línea]. http://www.milenio.com/negocios/Empresas-verdes-ganan-dineroBMV_0_308369381.html 30/07/2015.

Calva J. (2007). Sustentabilidad y desarrollo ambiental. Porrúa, México D.F., México, 302 pp.

Cemefi (2014). El concepto de responsabilidad social empresarial. Centro Mexicano de Filantropía [en línea]. http://www.cemefi.org/esr/images/stories/pdf/ esr/concepto_esr.pdf 20/05/2015. 
Chamorro A. (2001). La gestión medioambiental como una ventaja competitiva [en línea]. http://www.5campus. org/leccion/gestmed 02/06/2015.

TCNA (2014). Green squared certified products. Tile Council of North America [en línea]. http:// greensquaredcertified.com/certified-products.html 03/07/2015.

Dieleman H. y Juárez-Nájera M. (2008). ¿Cómo se puede diseñar educación para la sustentabilidad? Rev. Int. Contam. Ambie. 24, 131-147.

DJSI (2014). DSJI 2014 Review results. Dow Jones Sustainable Index [en línea]. http://www.sustainability-indices.com/images/DJSI_Review_Presentation_09_2014_final.pdf 15/06/2015.

Durán G. (2000). Medir la sostenibilidad: Indicadores económicos, ecológicos y sociales [en línea]. http:// pendientedemigracion.ucm.es/info/ec/jec $7 / \mathrm{pdf} / \mathrm{com} 1$ 6.pdf 17/06/2015.

Espinosa R. M., Turpin S., Vázquez R. C., Vázquez A., Cisneros A., De la Torre A. y García B. A. (2013). La gestión ambiental en una institución de educación superior asociada a las prácticas de separación y recuperación de residuos. Rev. Int. Contam. Ambie. 29, 49-57.

Garzón A. (1995). Gran diccionario enciclopédico visual. Programa Educativo Visual, Bogotá, Colombia, 1291 pp.

GlobalSTD (2014). Responsabilidad social. GlobalSTD Certification [en línea]. http://www.globalstd.com/ certificacion/responsabilidad-social 30/07/2015.

GSS (2004). Quality of life counts. Government Statically Service [en línea]. http://www.nies.go.jp/db/sdidoc/ qolc2004.pdf 11/05/2015.

Hernández R., Fernández C. y Baptista P. (2010). Metodología de la investigación. McGraw-Hill, México D.F., México, 320 pp.

Hoegen M. (2009). Statistics and the quality life [en línea]. http://www.oecd.org/site/progresskorea/globalproject/44227733.pdf 30/07/2015.

IMSS (2014). Ley del Seguro Social. Instituto Mexicano del Seguro Social [en línea]. http://www.imss.gob.mx/ sites/all/statics/pdf/LSS.pdf 24/07/2015.

Juárez-Nájera M., Rivera-Martínez J.G. y Hafkamp W.A. (2010). An explorative socio-psychological model for determining sustainable behavior: Pilot study in German and Mexican Universities. J. Clean. Prod. 18, 686-694. DOI: 10.1016/j.jclepro.2009.09.018
KPMG (2011). Encuesta: Desarrollo sostenible en México 2.0 [en línea]. http://www.kpmg.com/MX/es/ IssuesAndInsights/ArticlesPublications/Documents/ Estudios/2011/Encuesta-sostenibilidad-2011.pdf 29/05/2015.

López B. (2012). Ser sostenible o ser rentable. Revista Corrugando 29, 38-46

Lubin D. y Esty D. (2010). El imperativo de la sustentabilidad. Harvard Bus. Rev. 88, 36-46.

Mihelcic J. y Zimmerman J. (2012). Ingeniería ambiental. Fundamentos, sustentabilidad y diseño. Alfaomega, México D.F., México, 696 pp.

Montgomery D. (2014). Diseño y análisis de experimentos. 2a ed. Limusa Wiley, México D.F., México, 686 pp.

PROFEPA (2014). Programa nacional de auditoría ambiental México. Procuraduría Federal de Protección al Ambiente [en línea]. http://www.profepa.gob.mx/ innovaportal $/ \mathrm{v} / 26 / 1 / \mathrm{mx} /$ programa_nacional_de_auditoria_ambiental.html 18/07/2015.

Rincón E. y Wellens A. (2011). Cálculo de indicadores de ecoeficiencia para dos empresas ladrilleras mexicanas. Rev. Int. Contam. Ambie. 27, 333-345.

Salcedo M. P., Rebolloso F. y Barber C. (2010). El desarrollo sustentable. Modelo de conciliación entre el progreso económico, la justicia social y la preservación del medio ambiente. Gestión y estrategia 37, 17-31.

STPS (2014). Normas oficiales mexicanas. Secretaría del Trabajo y Previsión Social [en línea]. http://www.stps. gob.mx/bp/secciones/conoce/marco_juridico/noms. html 30/06/2015.

Sociedad Mexicana de Cerámica Zona Norte (2014). Localización de la industria cerámica en México [en línea]. http://www.soceramnorte.com.mx/mapa2.html 25/05/2015.

Stock D. (2014). World production and consumption of ceramic tiles. Tile Today 85, 54-63.

UN (1987). Report of the world commission on environment and development. United Nations [en línea]. http://www.un-documents.net/our-common-future. pdf 14/07/2015.

Vagias W. M. (2006). Likert-Type scale response anchors [en línea]. https://www.clemson.edu/centers-institutes/ tourism/documents/sample-scales.pdf 20/07/2015. 